\title{
Desarrollo autonómico de la prestación económica de asistencia personal
}

\author{
Fernando M. Jimeno Jiménez \\ UNED Ceuta \\ fernandom.jimeno@gmail.com
}

\begin{abstract}
Artikulu honetan, laguntza pertsonalerako prestazio ekonomikoaren lurraldeko arau-garapena aztertzen da. Autonomia sustatzeko programa da, eta egun gutxi ezartzen dena Espainiako mendetasunsisteman; baina etorkizun itxaropentsua du. Estatuko araudiak gutxieneko batzuk finkatzen ditu, eta autonomia-erkidegoen eskumena da edukian eta aintzatespen-eskakizunetan sakontzea; batez ere finantzaketa propioaren eremuan jarduten dutenean. Azken urteotan aurrera egin da gai hau esklusiboki araututa, elkarteek, bereziki Bizitza Independentearen Mugimenduak, eskatzen duten bultzadaren ondorioz. Egin beharreko zereginen definizioa, prestazioa aitortzera bideratutako zuzkidura ekonomiko handiagoa, eta bateragarritasun-eredu malguago eta kasu zehatzari egokituagoa izateko aukera, programa honen aurrerabidean garrantzitsuak diren elementuak antzematen dira.
\end{abstract}

\section{GAKO-HITZAK:}

Laguntza pertsonala, prestazio ekonomikoa, autonomia-sustapena, mendetasun-egoera, autonomia-erkidegoko arautegia.
En el presente artículo se analiza el desarrollo normativo territorial de la prestación económica de asistencia personal, programa de promoción de la autonomía con escasa implantación actual en el sistema de dependencia español, aunque con esperanzador futuro. La regulación estatal fija unos mínimos, siendo competencia de las comunidades autónomas profundizar en su contenido y los requisitos de reconocimiento, especialmente cuando actúan en el ámbito de financiación propio. En los últimos años se ha avanzado con la regulación en exclusividad de esta materia, circunstancia derivada del impulso que demandan las asociaciones, en especial el Movimiento de Vida Independiente. La definición de tareas a realizar, la mayor dotación económica destinada al reconocimiento de la prestación, así como la posibilidad de un modelo de compatibilidad más flexible y ajustado al caso concreto, se atisban elementos relevantes en el progreso de este programa.

\section{Palabras Clave:}

Asistencia personal, prestación económica, promoción autonomía, situación de dependencia, normativa autonómica. 


\section{Introducción}

En la primera década del siglo XXI, el poder legislativo español aprueba la regulación del sistema de autonomía personal y atención a las personas en situación de dependencia (LAPAD), coincidiendo en el tiempo con la ratificación de la Convención Internacional sobre los Derechos de las Personas con Discapacidad. Ambos instrumentos incluyen en su articulado contenido normativo básico y compromisos futuros, respectivamente, dirigidos a definir y potenciar la asistencia personal. El primero de los textos legales define la asistencia personal, indicando que se considera un servicio prestado (por el asistente personal), con el objetivo de fomentar la vida independiente de la persona en situación de dependencia, promoviendo o potenciando su autonomía personal, finalidad específica de la LAPAD (junto a la atención de la situación de dependencia). ¿Cómo actúa la figura del asistente personal? Colaborando en la realización o ejecutando las tareas propias de la vida ordinaria de la persona en situación de dependencia. A pesar de su novedad en la LAPAD, tiene largo recorrido en Estados Unidos y varios países europeos (Foro de Vida Independiente y Divertad, 2015).

La LAPAD debe ponerse en relación con la ratificación por el Estado español en 2008 de la Convención Internacional sobre Derechos de las Personas con Discapacidad, toda vez que las partes firmantes se comprometen y asumen el derecho a la vida independiente, garantizando la vida en comunidad en condiciones de igualdad, de modo que se evite la situación de aislamiento. En el texto internacional se produce una apuesta decidida por la asistencia personal en tanto que instrumento que favorece la capacidad de actuación autónoma y la libre toma de decisiones individuales. No obstante, se mantienen otras opciones de diferente naturaleza (atención domiciliaria, residencial u otros servicios de apoyo comunitario). En el reconocimiento y desarrollo de la asistencia personal ha resultado fundamental la actuación del Movimiento de Vida Independiente (Carbonell, 2017), aportando contribuciones no solo teóricas, sino también prácticas en su configuración y efectiva realización.

Muestra de la incidencia del contenido de la referida convención internacional es la reciente aprobación de la reforma de la legislación civil y procesal referida al apoyo a las personas con discapacidad en el ejercicio de su capacidad jurídica.

La atención que realiza el sistema de la LAPAD respecto de la asistencia personal adopta la forma de prestación económica. En un primer momento, se detecta que el contenido de esta norma en la materia de estudio es mínimo, orientado a establecer unas reglas comunes facilitadoras de un desarrollo autonómico amplio.

El objetivo del presente artículo pretende un análisis descriptivo, profundizar en los condicionantes establecidos en la normativa española, requisitos exigidos para el reconocimiento de la prestación económica de asistencia personal. Para ello, se analizarán las reglas nacionales establecidas en la LAPAD y su normativa de desarrollo, generalizadas a nivel estatal. Posteriormente se profundizará en el desarrollo territorial, reflexionando en torno a sus elementos particulares, así como en los intentos dirigidos hacia la mayor intensidad en la difusión del programa y aceptación por sus destinatarios.

Desde la entrada en vigor de la LAPAD, el desarrollo de la prestación económica de asistencia personal ha sido muy limitado. Después de catorce años desde la implantación del sistema de dependencia, es el programa individual de atención menos reconocido a nivel nacional (0,56\%). La excepción la representa el País Vasco (7,13\%) y, en menor medida, Castilla y León (1,05\%), siendo testimonial o inexistente en las restantes autonomías ${ }^{1}$.

Se puede comprobar que existe un importante desajuste entre las posibilidades potenciales de este recurso y la realidad (Carbonell, 2017), siendo necesarias su mayor difusión y conocimiento (Saavedra, 2018). Debe matizarse que hay estudios que demuestran el impacto positivo de un mayor desarrollo de la asistencia personal, toda vez que el retorno de la inversión es más elevado que el de otros programas (Quezada y Motos, 2017). De igual modo, es una garantía d el respeto a la voluntad de la persona con diversidad funcional (Suñe y Martínez, 2015).

\section{La prestación económica de asistencia personal en la ley de dependencia y normativa de desarrollo estatal}

El modelo de protección en la LAPAD se articula en torno a servicios y prestaciones económicas. Los primeros, facilitados por la Administración Pública competente con su red de servicios públicos y privados concertados, son considerados programas en especie y son prioritarios en el momento de asignar el programa individual de atención (PIA). Las prestaciones económicas implican una transferencia económica con destino variado, unas veces finalista (la asistencia personal o la vinculada al servicio), otras sin justificación, aunque orientadas a la adecuada atención del beneficiario (cuidados en el entorno familiar).

\subsection{Naturaleza de la prestación económica de asistencia personal}

El objetivo de la prestación económica de asistencia personal está orientado a contribuir a la contratación

${ }^{1}$ Información obtenida en la página oficial del Imserso (datos referidos a 30 de junio de 2021); 〈https://www.imserso.es/InterPresent1/groups/imserso/documents/binario/estsisaad20210630.pdf〉. 
de una asistencia personal, durante un número de horas, facilitadoras del acceso al trabajo, la educación y una vida más autónoma en el ejercicio de las actividades básicas de la vida diaria. En esta definición que facilita la LAPAD (artículo 19) deben tomarse en consideración dos aspectos relevantes. Por un lado, concreta un destino último (facilitar trabajo y educación), aunque deja abierta la posibilidad de realizar otras actuaciones, aquellas que redunden en una vida más independiente de la persona. Por otro lado, el objetivo de la prestación económica de asistencia personal es "contribuir”, en definición de la Real Academia Española, “ayudar, junto a otros, a la consecución de un fin", un apoyo que favorezca el desarrollo individual en la vida ordinaria.

La asistencia personal tiene formato de prestación económica en tanto que transferencia monetaria. Como contrapartida, el interesado asume la justificación de la contratación efectuada y la acreditación de su coste (la atención prestada debe superar, o al menos ser igual, a la financiación pública reconocida). La necesidad de demostrar el gasto efectuado permite considerar su naturaleza como finalista (Jimeno, 2021), con una aproximación al concepto de servicio, en denominación del consejo territorial del sistema de dependencia, servicio indirecto (Secretaría de Estado de Servicios Sociales e Igualdad, 2014). Este planteamiento encaja con la profesionalización y titulación exigible al asistente personal, aunque, al fundamentarse en una relación personal tan directa entre beneficiario y asistente, sea necesario adicionalmente un elevado nivel de empatía, elemento básico (Fariñas, 2018).

Debe diferenciarse de la prestación económica vinculada al servicio (artículo 17 de la LAPAD), cuya finalidad es el acceso al mercado privado ante ausencia de servicio público o privado concertado en el catálogo, coincidiendo en la naturaleza finalista y la necesidad de justificación. Se diferencian ante el carácter sustitutivo de la vinculación (no existe servicio público o concertado) y la necesaria contratación con entidad debidamente acreditada (artículo 14.3 de la LAPAD), frente al fomento de la vida independiente y la promoción de una vida más autónoma en la asistencia personal, efectuada con persona cualificada en virtud de contrato laboral o entidad prestadora de servicios.

El deslinde respecto de la excepcional prestación económica para cuidados en el entorno familiar y apoyo a cuidadores (artículo 18 de la LAPAD) es nítido, por justificarse su reconocimiento en las atenciones informales efectuadas por el núcleo familiar más cercano (hasta tercer grado) o persona del entorno, en su caso. No existe obligación alguna de justificar su destino, aunque se pueda realizar seguimiento para comprobar la adecuación de los cuidados prestados.

Por último, es necesario diferenciar la asistencia personal del servicio de ayuda a domicilio. Las atenciones domiciliarias (artículo 23 de la LAPAD), en su doble vertiente de cuidados versus tareas domésticas, deben ser efectuadas por entidades debidamente acreditadas, con programa definido de actuación, tomando como referencia la vivienda de la persona en situación de dependencia ${ }^{2}$. Por tanto, la ayuda a domicilio pivota en torno a la residencia habitual del beneficiario y la asistencia personal se define por su finalidad, la promoción de la autonomía y el desenvolvimiento en comunidad. No obstante, las tareas que debe desarrollar la asistencia son complejas de definir y relacionadas con múltiples áreas de actuación (Rodríguez-Picavea, 2007) ${ }^{3}$.

\subsection{Normativa estatal}

La regulación estatal de la prestación económica de asistencia personal se localiza en la propia LAPAD (fundamentalmente en sus artículos 14, 19 y 20), en el reglamento sobre prestaciones (Real Decreto 1051/2013) y las propuestas con alcance limitado que formule el Consejo Territorial de Servicios Sociales y del Sistema para la Autonomía y Atención a la Dependencia. Podría considerarse como una normativa mínima, con finalidad homogeneizadora, aunque limitada en su alcance, dada la tendencia al desarrollo autonómico. Desde el movimiento asociativo (Foro de Vida Independiente y Divertad, 2015), junto a investigaciones publicadas, se demanda una regulación de mayor intensidad (Fariñas, 2018; Bañez y Comas, 2021).

Dentro del respeto a la normativa estatal fijada en la LAPAD y el reglamento sobre prestaciones, la competencia para su desarrollo es autonómica (la Administración General del Estado en las Ciudades Autónomas de Ceuta y Melilla). Debe indicarse que, en la regulación nacional de la prestación de asistencia personal, deben diferenciarse las reglas de alcance obligatorio frente a las sugeridas por el consejo territorial.

\subsubsection{Reglas de contenido necesario fijadas en la LAPAD}

Entre las normas de contenido básico, se han analizado en páginas anteriores el concepto y la naturaleza finalista de la prestación económica. Acerca de esta última apreciación, es preciso matizar la libertad de alternativas de que dispone el beneficiario para contratar, bien con una entidad (con o sin ánimo de lucro), bien una relación laboral o la compleja situación de la relación jurídica con empresario individual (persona física), situación esta que puede originar problemas en su deslinde, análisis que se efectuará en páginas posteriores.

\footnotetext{
${ }^{2}$ Las actuaciones del servicio de ayuda a domicilio tienen lugar en el hogar, entorno habitual de la persona en situación de dependencia, aunque puedan producirse salidas al exterior (por ejemplo, acompañamientos para realizar gestiones, ocio y tiempo libre).

${ }^{3}$ Rodríguez-Picavea (2007) identifica las siguientes áreas de actuación de la asistencia: personales, hogar, acompañamiento, conducción, comunicación, coordinación, excepcionales y especiales.
} 
La normativa estatal determina en el artículo 31 de la LAPAD la obligatoria deducción (se utiliza expresamente la denominación "deducirá") sobre las prestaciones económicas del sistema de dependencia, entre las que se incluye la asistencia personal, de aquellos complementos identificados como de análoga naturaleza y finalidad previstos en los regímenes públicos de protección social, señalando de manera concreta los complementos de gran invalidez; de asignación económica por hijo a cargo mayor de dieciocho años y con al menos un $75 \%$ de discapacidad; de tercera persona en las pensiones no contributivas de invalidez; y el subsidio por el mismo concepto establecido en la Ley de Integración Social de los Minusválidos (Ley 13/1982). Debe indicarse que no es una enumeración cerrada, abierta a otras concreciones, cuya finalidad es evitar el solapamiento entre prestaciones (Jimeno, 2018). Una vez reconocida la prestación económica, es inembargable (artículo 14.8 de la LAPAD), salvo lo previsto en el artículo 608 de la Ley de Enjuiciamiento Civil, que hace referencia a la ejecución de sentencias condenatorias al pago de alimentos.

La cuantía de la prestación económica de asistencia personal prevista en la LAPAD es competencia estatal, en virtud de Real Decreto del Gobierno. Los importes máximos permanecen congelados desde $2012(715,07$ euros mensuales para el grado III, 426,12 euros al mes en el grado II y 300 euros al mes para el grado I), cifras que pueden minorarse en función de la capacidad económica de la persona dependientes o de los complementos de análoga naturaleza y finalidad, incluso dejando sin contenido económico la resolución final, circunstancia que justifica el escaso éxito obtenido por esta modalidad. La posibilidad de acrecentar el montante por las comunidades autónomas con cargo a sus presupuestos (artículo 11.2 de la LAPAD), es una opción que desarrollan algunas autonomías, medida amparada en su nivel adicional de protección y punto clave junto a otras consideraciones para activar su reconocimiento en mayor escala.

La aprobación de la prestación económica de asistencia personal es consecuencia de la confección de un programa individual de atención (artículo 29 de la LAPAD), dado que el órgano administrativo entiende que es la modalidad de intervención adecuada a las necesidades del beneficiario, una vez consultado el interesado o su familia. Una innovación introducida en el año 2012 (Real Decreto Ley 20/2012, artículo 22.7) es la aplicación de la asistencia personal a todos los grados de situación de dependencia, moderada (I), severa (II) y gran dependencia (III), ya que hasta ese momento estaba reservada solamente para el último. Por tanto, la asignación del programa es viable para cualquier persona en situación de dependencia, una vez que se determine en el programa individual la idoneidad para la promoción de la autonomía.

Una última apreciación es la preferencia de los servicios en la configuración del programa individual de atención (artículo 14.2 de la LAPAD), condicionante que parece estar referido a la excepcionalidad de la prestación económica para cuidados familiares (artículo 14.4 de la LAPAD) y no tanto respecto de la asistencia personal, posiblemente debido a su configuración como recurso vinculado a la promoción de la autonomía (artículo 14.5 de la LAPAD).

\subsubsection{Las aportaciones del Consejo Territorial de Servicios Sociales y del Sistema para la Autonomía y Atención a la Dependencia}

Los acuerdos del consejo territorial aportan una visión de conjunto, un marco uniforme, una propuesta de actuación similar en todas las comunidades autónomas. En relación con la prestación de asistencia personal, han sido dos las materias afectadas. Por un lado, en el Acuerdo del Consejo Territorial de 13 de julio de 2012 se determina la cuantía de la prestación de asistencia personal en función del coste del servicio y de la capacidad económica del beneficiario (Secretaría de Estado de Servicios Sociales e Igualdad, 2012), sin que el contrato suscrito con el asistente personal tenga importe inferior a la cantidad reconocida; por otro, en el de 25 de noviembre de 2008 se establece la titulación mínima exigible al asistente personal (Secretaría de Estado de Política Social, Familias y Atención a la Dependencia y la Discapacidad, 2008), indicando la cualificación profesional de atención sociosanitaria a personas en domicilio, certificaciones de profesionalidad y equivalencias, junto a nuevas posibilidades como la habilitación provisional o excepcional ${ }^{4}$.

Sin embardo, debe tenerse en cuenta que la sentencia de la Audiencia Nacional de 25 de febrero de 2011 considera que los citados acuerdos del consejo territorial solamente tienen capacidad de propuesta, una invitación homogeneizadora a las comunidades autónomas en el momento de elaboración normativa. En este sentido, el propio acuerdo del consejo territorial de 13 de julio de 2012, al fijar los criterios mínimos comunes ( $n$ 으 11), determina que en seis meses las comunidades autónomas (Administraciones Públicas competentes) promoverán las disposiciones necesarias para dar cumplimiento y en el plazo de un año las adaptaciones sobre las situaciones jurídicas preexistentes.

\subsubsection{El régimen de incompatibilidad previsto en el reglamento de prestaciones del sistema de dependencia}

Por último, el reglamento de prestaciones (Real Decreto 1051/2013, art. 16) establece el régimen

\footnotetext{
${ }^{4}$ Se han previsto convocatorias públicas de habilitación provisional y excepcional, en función de ciertos requisitos recogidos en el Acuerdo del Consejo Territorial de 19 de octubre de 2017, así como durante la situación de pandemia derivada de la covid-19 en virtud del acuerdo de 23 de marzo de 2020.
} 
de incompatibilidades. Como regla general, la prestación de asistencia personal es incompatible con otras prestaciones económicas y con los servicios del catálogo, con excepción de los servicios de prevención, promoción de la autonomía personal y teleasistencia. No obstante, las Administraciones Públicas competentes puedan aprobar normativa propia que compatibilice la asistencia personal con los servicios de ayuda a domicilio, centro día/noche, prestación económica para cuidados en el entorno familiar y apoyo a cuidadores no profesionales. En todo caso, con su nivel de financiación adicional podrán establecer las comunidades autónomas su propio régimen de compatibilidad.

\section{Análisis del desarrollo normativo autonómico}

Como se ha indicado anteriormente, se pueden establecer como reglas de contenido esencial: el concepto de asistencia personal como programa de promoción de la autonomía; la naturaleza finalista de la prestación económica; la libre contratación de servicios o laboral; la determinación de la cuantía de las prestaciones por medio de Real Decreto del Gobierno; el carácter deducible de las prestaciones de idéntica naturaleza y finalidad, el reconocimiento en virtud de aprobación del programa individual de atención; la extensión a los tres grados de situación de dependencia; la inembargabilidad de las prestaciones económicas (con alguna excepción) y la prioridad de los servicios del catálogo. Pueden asumirse a nivel autonómico los acuerdos aprobados por el consejo territorial en materia de participación en el coste del servicio y sobre titulación del asistente/a personal, así como adaptar la habilitación establecida sobre régimen de incompatibilidades.

Una vez tomadas en consideración las reglas anteriores, las Administraciones Públicas competentes establecen sus regulaciones dentro de su marco competencial. En las siguientes páginas se analizan elementos comunes y diferenciales.

\subsection{Rango y marco normativo}

El rango normativo donde se ha establecido la regulación básica de la prestación de asistencia personal ha sido diverso. Unas veces, el decreto del consejo de gobierno (Illes Balears, Castilla la Mancha, Galicia, Madrid, Murcia o Valencia); en otras ocasiones, la orden de la consejería que asume las funciones (Andalucía, Aragón, Castilla y León, Extremadura, La Rioja o territorio Imserso). Finalmente, el decreto foral (Álava, Gipuzkoa y Bizkaia) 5 .

${ }^{5}$ En algún supuesto, ha sido una norma con rango de ley la que regula determinados aspectos (régimen de incompatibilidades en la disposición adicional 13 de la ley de servicios sociales de Canarias).
En los últimos años, bajo la tendencia de facilitar mayor promoción, han sido varias las comunidades autónomas que han aprobado normativa en exclusiva sobre la asistencia personal (Cataluña, Navarra o Diputación Foral de Bizkaia), o aportando instrucciones específicas (Valencia). La regla general ha sido la redacción de una normativa genérica, en el marco de los servicios y las prestaciones económicas del sistema de dependencia (Andalucía, Asturias - Castilla la Mancha), con inclusión adicional de la capacidad económica (Illes Balears, Cantabria o Castilla y León). Las diputaciones forales de Álava y Gipuzkoa se centran en las prestaciones económicas y en la Xunta de Galicia en la cartera de servicios. La Comunidad de Madrid y el Imserso (Ceuta y Melilla) en la regulación del reconocimiento del derecho a la situación de dependencia y las prestaciones del sistema.

\subsection{Objetivo de la asistencia personal}

El modelo autonómico más extendido es el que relaciona la asistencia personal con la promoción de la autonomía en actividades básicas (Aragón, Cantabria, Extremadura, La Rioja o Madrid).

Algunas comunidades autónomas matizan que el objetivo está destinado a contribuir a los gastos de contratación del asistente personal, facilitando la educación y el trabajo (Andalucía, Asturias, Castilla y León, diputaciones forales de Álava y Gipuzkoa), tomando como referencia temporal un número de horas diarias.

De manera concreta, se incide en considerar la asistencia personal como instrumento destinado a favorecer la participación en asociaciones (Asturias) y a facilitar el acceso a recursos del entorno comunitario (Castilla la Mancha), así como la inclusión y participación en comunidad en los términos establecidos en la Convención de Naciones Unidas sobre los derechos de las personas con discapacidad (Comunidad Valenciana), de manera profesional y participando en actividades educativas y laborales de manera regular (Cantabria).

En Cataluña, tras consulta ciudadana ${ }^{6}$, se precisa que la asistencia personal se destina a la ayuda en el ejercicio de la autodeterminación (habilidad para definir y conseguir objetivos propios) y fomentar la autonomía personal, la integración social y la participación en la vida comunitaria. De igual forma, la futura normativa de la Generalitat pretende impulsar la asistencia personal para personas con discapacidad física y sordoceguera, con un plan piloto para las personas con discapacidad intelectual (La Vanguardia, 2020).

${ }^{6}$ La iniciativa obedece al proyecto de regular la asistencia per sonal, consultando los colectivos que pueden acceder a su reconocimiento, la intensidad e importe de la prestación económica, la formación del asistente, con adaptación a la diversidad funcional, y la incidencia en la reducción de listas de espera residenciales. 


\subsection{Requisitos de acceso a la prestación económica}

Se debe analizar el cumplimiento de requisitos desde una doble perspectiva, los exigidos a la persona en situación de dependencia y los que debe reunir el asistente personal.

\subsubsection{Requisitos exigibles a la persona en situación de dependencia}

Con independencia de los requisitos generales establecidos por la LAPAD (encontrarse en situación de dependencia en cualquiera de los tres grados y residencia en territorio español y haberlo hecho durante cinco años, de los cuales dos deben ser inmediatamente anteriores a la solicitud), y su reconocimiento como programa individual de atención, las comunidades autónomas establecen ciertas condiciones susceptibles de interés.

Con carácter general, se exige capacidad en el interesado para determinar los servicios necesarios, capacidad de control e impartición de instrucciones al asistente personal, directamente o por medio de representante (Andalucía, Illes Balears, Castilla la Mancha, Extremadura o La Rioja).

De manera concreta, las tareas a ejecutar deben quedar definidas y planificadas en función de las necesidades de apoyo, contenidas en documento escrito (Castilla la Mancha). La persona en situación de dependencia organiza el servicio según sus preferencias, decide la provisión y supervisa su prestación (Cataluña).

Algunas comunidades autónomas regulan requisitos específicos para reconocer el servicio. Así, presentar un proyecto de vida independiente ${ }^{7}$ (Comunidad Valenciana, Navarra ${ }^{8}$, diputaciones forales de Álava, Gipuzkoa y Bizkaia) o haber cumplidos tres años de residencia (Comunidad Valenciana ${ }^{9}$, Navarra, diputaciones forales de Álava y Gipuzkoa), siempre que suponga beneficio al desarrollo personal, laboral, social y comunitario. En Bizkaia ${ }^{10}$ se determina como requisito de edad haber cumplido 16 años con emancipación o ser mayor de 18 y menor de 65 años, salvo que al cumplir esta edad ya fueran beneficiarias, en cuyo caso continuará el reconocimiento.

La Diputación Foral de Bizkaia exige a las personas en situación de dependencia con grado I y

\footnotetext{
7 En Navarra el plan individual de vida independiente se realiza por medio de la Oficina de Vida Independiente. En la Comunidad Valenciana existe un modelo preestablecido, donde se recogerán las características y condiciones del plan de vida independiente.

${ }^{8}$ Navarra considera la prestación de asistencia personal como garantizada para personas en situación de dependencia y no garantizada para personas con discapacidad igual o superior al $65 \%$.

${ }^{9}$ La Generalitat Valenciana diferencia las tipologías en función de la edad: terapéutica para menores de 18 años (impulso de capacidades y fortalezas en la primera etapa vital), personas entre 18 y 64 años, y mayores de 65 años.

${ }^{10}$ En Bizkaia el plan individual de vida independiente debe describir la situación de convivencia, las actividades ocupacionales, las de participación comunitaria y el número de horas necesarias, detallando puntualmente las funciones y tareas a realizar.
}

puntuación ubicada entre 25 y 39 puntos, de modo adicional, encontrarse valoradas con discapacidad igual o superior al $33 \%$ para acceder a la prestación económica. La Diputación Foral de Álava determina que para el acceso al nivel adicional de protección se exige empadronamiento y residencia efectiva en el Territorio Histórico de Álava durante tres años consecutivos inmediatamente anteriores a la primera solicitud de reconocimiento, o quienes reúnan el mismo periodo aunque con posterioridad a la resolución y, en todo caso, con una residencia mínima de ocho meses al año.

\subsubsection{Requisitos exigibles al asistente personal}

La regulación más representativa es la que exige al asistente personal los requisitos de mayoría de edad y residencia legal, al amparo de alguna fórmula contractual (relación jurídica con entidad social, empresa o contrato laboral), así como idoneidad para prestar los servicios (Andalucía, Illes Balears, Cantabria, Cataluña, La Rioja o Murcia).

De manera concreta, se matiza que no pueden acceder a la condición de asistente personal el cónyuge (pareja de hecho) y los parientes por consanguinidad, afinidad o adopción hasta el tercer grado de vínculo con la persona beneficiaria (Aragón, Asturias, Castilla La Mancha, Extremadura, Madrid, diputaciones forales de Álava y Gipuzkoa), un criterio relevante para diferenciar esta prestación económica respecto de la que protege los cuidados para el entorno familiar (y apoyo a cuidadores familiares), que sí exige esa relación (o con persona del entorno, en su caso). En determinadas autonomías la prohibición se extiende a la condición de tutor o acogedor (Navarra, diputaciones forales de Álava y Gipuzkoa). En la regulación de la Comunidad Valenciana, no es posible hasta el cuarto grado de consanguinidad o afinidad. En el decreto foral de Gipuzkoa no pueden acogerse a esta modalidad quienes pertenezcan a la misma orden religiosa y compartan ámbito residencial.

En la determinación de los requisitos de residencia, la Comunidad Valenciana exige empadronamiento en la Comunidad Autónoma para el asistente personal y en el decreto foral de Gipuzkoa, residir legalmente en el Estado español o francés.

En relación con la capacidad de la persona que pretenda ejercer la asistencia, en algún supuesto es preciso informe técnico acerca de su idoneidad (La Rioja), exigiendo su ratificación por los servicios sociales (Comunidad Valenciana) en el primer informe de seguimiento, conforme al tipo de diversidad funcional.

\subsection{Modalidades y exigencias en la contratación}

Es un principio básico la libertad de contratación de los servicios por la persona interesada (con los 
apoyos previstos tras la reciente reforma civil y procesal). Se pueden establecer varias posibilidades de contratación, con entidades sociales, empresas de servicios, empresario individual (autónomo) y relación sometida al régimen laboral. Las normativas autonómicas exigen de manera generalizada la acreditación en las primeras y requisitos de titulación para los trabajadores por cuenta propia y ajena. Ocupan una posición relevante las entidades sociales, en especial el modelo desarrollado por las oficinas de vida independiente, creadas por las personas con diversidad funcional y fundamentadas en un proyecto cuyo centro de atención son los beneficiarios, sin intervención de terceros (Gómez, Ordax, Moya y Sánchez, 2015).

Resulta fácil diferenciar la línea que separa un contrato de trabajo (relación laboral entre beneficiario y asistente) respecto de la contratación con una empresa dedicada a prestar específicamente a servicios asistenciales. Sin embargo, pueden surgir dudas en la relación con un trabajador por cuenta propia o autónomo. En este sentido, la jurisprudencia es nítida en sus conclusiones, al indicar que se deben tener en cuenta todas las circunstancias del caso en el momento de calificar su naturaleza (por cuenta propia o ajena), en particular si se reúnen las notas de ajenidad, dependencia, trabajo y retribución propias del contrato de trabajo, sometido a la legislación laboral, conforme a la sentencia de 26 de noviembre de 2012 del Tribunal Supremo.

Es importante destacar que en el contrato suscrito se deben fijar las directrices y cláusulas de confidencialidad (Illes Balears). En ningún supuesto la organización pública gestora será responsable, ni formará parte de la relación contractual establecida entre beneficiario y asistente (Cataluña). La Diputación Foral de Bizkaia establece un número mínimo de horas semanales a contratar, en función del grado de situación de dependencia reconocido (tres, cuatro o cinco, horas para los grados I, II y III, respectivamente).

Para finalizar este apartado, cabe reflejar la opinión del profesor Maldonado Molina (2015), al considerar que la modalidad contractual que mejor se adapta al concepto de vida independiente es el arrendamiento de servicios, por corresponderse con una relación jurídica entre iguales.

\subsubsection{Titulación exigible al asistente personal}

En páginas anteriores se ha comentado que el consejo territorial propone que las comunidades autónomas desarrollen normativa acerca de la cualificación profesional de atención sociosanitaria a personas en domicilio y equivalencias ${ }^{11}$,

${ }^{11} \mathrm{~A}$ modo de ejemplo, en el artículo 12 de la orden EMP 48/2009 en la Comunidad Autónoma de Cantabria se establecen como titulaciones habilitantes "la cualificación profesional de Atención Sociosanitaria a Personas en el Domicilio creada por el Real Decreto certificaciones de profesionalidad y fórmulas de habilitación provisional o excepcional, titulaciones requeridas para acceder a la prestación económica. La exigencia de titulación y formación está en línea con la idea de profesionalización, un perfil necesario para el desenvolvimiento de una persona con diversidad funcional (Rodríguez-Picavea, 2007).

Como muestra, la Comunidad de Cantabria toma en consideración la formación, experiencia y titulación, mientras que en la Comunidad Valenciana se valoran los servicios prestados en la atención de la situación de dependencia y la promoción de la autonomía, con titulaciones ${ }^{12}$ (equivalencias) ajustadas a las específicas necesidades de la persona y su edad (Diputación Foral de Álava).

Ante las dificultades de obtención de titulación entre quienes ya se encuentren prestando servicios, se han previsto fórmulas transitorias, como la habilitada en Castilla y León, al entender cumplido el requisito siempre que la antigüedad laboral supere los seis meses o, en su caso, se formalice compromiso de formación futura.

\subsubsection{Acreditación de empresas de servicios}

Si la opción elegida por el beneficiario ha sido contratar con una entidad privada, en virtud de contrato de servicios, de naturaleza no laboral, se ha previsto en la LAPAD la acreditación como instrumento dirigido a examinar los requisitos mínimos y estándares de calidad asistenciales (Jimeno, 2017). La acreditación es una exigencia recogida expresamente en la normativa autonómica (Asturias, Cataluña, Madrid o Murcia); específicamente en la Comunidad de Castilla y León es necesario que el servicio de asistencia personal esté inscrito en el registro de entidades, servicios y centros de carácter social, bien sean entidades de iniciativa social, bien inscritas en el registro mercantil (Cataluña). En la Diputación Foral de Bizkaia es necesaria la inscripción de la entidad prestadora en el Registro Foral de Servicios Sociales.

295/2004, de 20 de febrero, o de Atención Sociosanitaria a Personas Dependientes en Instituciones Sociales, creada por el Real Decreto 1368/2007, de 19 de octubre, así como los correspondientes certificados de profesionalidad regulados por el Real Decretos 1379/2008, de 1 de agosto o por las vías equivalentes que se determinen. Asimismo, se considerarán los títulos de Técnico en Cuidados Auxiliares de Enfermería establecido por el Real Decreto 546/1995, de 7 de abril, o Técnico de Atención Sociosanitaria, establecido por el Real Decreto 496/2003, de 2 de mayo.

${ }_{12}$ Títulos de Técnico/a en Cuidados Auxiliares de Enfermería, Técnico/a de Atención Sociosanitaria, Técnico/a de Atención a Personas Dependientes, Técnico/a en Integración Social, Mediación Comunicativa, Animación Sociocultural o Educación Infantil, Titulaciones superiores a las mencionadas en los apartados anteriores en la rama de Ciencias de la Salud, de la Educación y Sociales, Certificado de Profesionalidad de Atención Sociosanitaria a Personas Dependientes en Domicilio, Certificado de Profesionalidad de Atención Sociosanitaria a Personas Dependientes en Instituciones Sociales, Certificado de Profesionalidad de Inserción Laboral de Personas con Discapacidad. 


\subsection{Definición de tareas a realizar en la asistencia personal}

Las actuaciones y funciones del asistente personal deben estar enfocadas a fomentar la vida independiente, promoviendo o potenciando la autonomía personal. En este sentido, las comunidades autónomas han descendido a un nivel más concreto de detalle en su definición.

En la Orden ASC/471/2010 de Cataluña se precisa que la tarea principal es proporcionar el apoyo necesario para superar las limitaciones funcionales y colaborar en las tareas de la vida cotidiana para potenciar la autonomía personal y fomentar la vida independiente. No son tareas propias del asistente personal las derivadas de limpieza en profundidad, mantenimiento o mejora del domicilio, ni las que requieran formación en el ámbito sanitario. Sí son funciones incluidas en la asistencia el mantenimiento de la higiene y orden del espacio doméstico relacionadas con la atención personal. En todo caso, los cometidos que deban realizarse deben estar referidos al cumplimiento de la relación contractual y los de orden ético en el marco del código deontológico. Si se efectúan acciones de autocuidado que realiza el beneficiario o un familiar, el asistente debe contar con formación previa instrumental, asumiendo el beneficiario la responsabilidad por escrito.

La Orden Foral 224/2019 en Navarra, determina que, en función de las necesidades de la persona beneficiaria, podrán realizar los asistentes personales funciones de apoyo en: higiene, alimentación, mantenimiento de la salud y autocuidados; tareas domésticas; desplazamientos; comunicación; contexto laboral; formación profesional; asistencia a la enseñanza superior e investigación; contextos culturales, ocio y deporte; mejora de la empleabilidad; participación comunitaria y ciudadana; y actividades especiales mutuamente acordadas.

El Decreto 44/2015 de la Diputación Foral de Bizkaia refleja las funciones en el marco del plan individual de vida independientes, incluyendo: tareas personales (aseo, vestido, ayuda alimentación, transferencias, movilizaciones y ayuda necesidades fisiológicas); acompañamiento y asistencia; conducción, donde el importe destinado a las primeras no puede representar el $75 \%$ o más de las horas. Se declaran excluidas: las relacionadas con la comida (preparación y compra); relacionadas con el vestido (lavado, planchado, ordenación, repaso, adquisición) o con el mantenimiento del hogar.

\subsection{Obligación de colaborar por el beneficiario}

En las normas autonómicas se recogen menciones expresas al deber de colaboración que asume la persona en situación de dependencia (representación, en su caso), referido en esencia a facilitar información y comunicar modificaciones que afecten a la situación de reconocimiento, así como a destinar las prestaciones económicas a su finalidad y justificar la aplicación (Illes Balears) o facilitar el seguimiento público del cumplimiento de los requisitos (Castilla La Mancha, Extremadura), con especial incidencia en aquella documentación que no obre en poder de las Administraciones Públicas (La Rioja). De igual modo, comunicar cualquier incidencia que afecte al reconocimiento o produzca una modificación (Diputación Foral de Álava), de manera especial las referidas al asistente personal (Galicia).

Algunas comunidades autónomas establecen preceptos más específicos con la intención de profundizar en el contenido obligacional. Así, destinar la prestación a la finalidad prevista y someterse a actuaciones de comprobación (Navarra), o autorizar el ingreso domiciliario a efectos de seguimiento (Diputación Foral de Álava). La Diputación Foral de Gipuzkoa extiende la colaboración a la información sobre reintegro de prestaciones, la obtención del documento nacional de identidad (por cumplimiento de catorce años) o la adquisición de nacionalidad española. La Diputación Foral de Bizkaia precisa que las comunicaciones deben efectuarse dentro del plazo de un mes desde que se produzcan, especialmente las que afecten a una modificación del programa de vida independiente.

Finalmente, reflejar que los beneficiarios deben destinar la prestación a su finalidad, aunque en Castilla y León pueden justificar su contenido con otros servicios reflejados en el programa individual de atención.

\subsection{Importe de la prestación económica}

Se aprecian diferencias relevantes en la cantidad a reconocer en función de cada autonomía. Por una parte, se encuentran las comunidades autónomas que utilizan las referencias económicas establecidas en el Real Decreto Ley $20 / 2012^{13}$; por otra, aquellas que reconocen mayores importes en base al nivel de financiación propio ${ }^{14}$.

Es rasgo común definidor fijar un importe máximo según el grado de situación de dependencia, condicionando la resolución final en función de la capacidad económica, la intensidad de la dedicación horaria y el coste del servicio. La capacidad económica actúa como componente reductor, unas veces tomando en consideración únicamente las

${ }^{13}$ Gran dependencia, 715,07 euros mensuales; dependencia severa, 426,12 euros/mes y la dependencia moderada, 300 euros/mes.

${ }^{14}$ A modo de ejemplo, la Orden Foral 224/2019 del Consejero de Derechos Sociales de Navarra: gran dependencia, un máximo de 1.600 euros y un mínimo de 1.280 euros; dependencia severa, un máximo de 1.600 euros y un mínimo de 1.280 euros; dependencia moderada, un máximo de 700 euros y un mínimo de 560 euros. La discapacidad superior al $65 \%$, un máximo de 1.600 euros y un mínimo de 1.280 euros. Las cuantías se actualizan de conformidad con el IPC. 
rentas personales del beneficiario (por ejemplo, Andalucía o el denominado territorio Imserso), otras en función de los rendimientos familiares divididos por el número de miembros (Navarra). De modo general, las escalas unas veces aplican un porcentaje de reducción en función del Indicador Público de Renta de Efectos Múltiples (Andalucía o Diputación Foral de Gipuzkoa), y otras la referencia es el salario mínimo interprofesional (Diputación Foral de Gipuzkoa). La intensidad se calcula conforme a la dedicación horaria, de modo que con la intensidad completa se podría acceder a la prestación íntegra y con la parcial de manera proporcional.

La reglamentación autonómica extiende el abono de la prestación económica durante doce mensualidades anuales, abonables por medio de transferencia bancaria a mes vencido ${ }^{15}$, debiendo destinarse su totalidad a la finalidad prevista. La Diputación Foral de Álava indica que la prestación económica es intransferible, no puede ofrecerse en garantía de obligaciones, ni objeto de cesión (total o parcial) ${ }^{16}$, compensación o descuento, así como retención o embargo.

Por último, no siempre se reconoce una prestación económica, admitiéndose la posibilidad de reconocimiento directo del servicio proporcionado por una entidad acreditada (Cataluña ${ }^{17}$ ).

\subsection{Régimen de incompatibilidad}

De acuerdo con lo previsto en el Reglamento de Prestaciones (artículo 16 del Real Decreto 1051/2013), las comunidades autónomas reflejan en su normativa la compatibilidad de las prestaciones de asistencia personal con los servicios de prevención, promoción de la autonomía personal y teleasistencia. Determinadas autonomías, haciendo uso de la habilitación prevista, así como con cargo a su nivel adicional, han establecido reglas propias.

En Andalucía es compatible la asistencia personal con los servicios de ayuda a domicilio, centro de día/noche y atención residencial durante el periodo vacacional del asistente. Asturias facilita el ingreso residencial temporal del beneficiario por un periodo máximo de treinta días al año (centro público o privado concertado). En Castilla y León se matiza la compatibilidad con la teleasistencia básica y avanzada. La Comunidad Valenciana excluye expresamente de la compatibilidad a las viviendas tuteladas. En Navarra se admite la compatibilidad con la atención diurna, la residencial y el servicio domiciliario municipal.

${ }^{15}$ En los primeros diez días del mes siguiente (Cantabria).

${ }^{16}$ Salvo para el reintegro de cantidades indebidamente percibidas o deuda pendiente de pago al Instituto Foral de Bienestar Social, se refleja en el Decreto Foral (Diputación Foral de Álava).

${ }_{17}$ La orden reguladora establece la prestación económica para las personas con gran dependencia y el servicio en los supuestos de dependencia severa y moderada.
Cataluña ha previsto un modelo de incompatibilidad entre prestaciones que tengan el mismo objeto y finalidad, admitiendo la posibilidad de utilizar los servicios de tecnologías de apoyo y cuidados, los comunes para personas con discapacidad, las ayudas técnicas y otras prestaciones de apoyo a la accesibilidad y supresión de barreras. No obstante, el catálogo de actuaciones del Sistema Catalán de Autonomía y Atención a la Dependencia (y del Sistema Público de Servicios Sociales en Cataluña) ha previsto la compatibilidad entre dos servicios o entre un servicio y una prestación económica, limitada por criterios de intensidad, indicando qué prestación es la principal y cuál la secundaria, fijando como regla general la primacía del servicio.

La Diputación Foral de Álava, después de indicar la compatibilidad entre un máximo de dos servicios/ prestaciones económicas para la dependencia grado I y un límite de tres para los grados II y III, establece reglas propias con cargo a su nivel de financiación propio 0 adicional. Así, para el grado I con 40 puntos o más de baremo (BVD), la asistencia personal será compatible con el centro de día en cualquier modalidad, con el centro nocturno para personas mayores, y con los servicios de respiro de centro diurno, nocturno y alojamiento para personas mayores y de vivienda con apoyos para personas con discapacidad o enfermedad mental (hasta 45 días anuales). Para los grados II y III, reglas similares, incluyendo el servicio de respiro en servicio residencial.

La Diputación Foral de Gipuzkoa establece la compatibilidad de la asistencia personal con la atención diurna, la ocupacional, estancias temporales (dos meses cada año continuos o discontinuos) y el centro sociosanitario, hospital psiquiátrico o similares (dos meses cada año, continuos o no), fijando minoraciones económicas por este motivo.

La Diputación Foral de Bizkaia declara la compatibilidad con el servicio de atención diurna, ayuda a domicilio, atención residencial inferior a dieciséis horas, estancia temporal residencial e ingreso sociosanitario limitado en el tiempo.

\subsection{Requisitos formales a justificar}

La justificación es la obligación asumida tras el abono de la prestación económica, aportando facturas y documentación contractual. Es regla común el deber de aportar los contratos derivados de la relación jurídica entre beneficiario y asistente (entidad social, servicios, autónomo o contrato laboral) y las facturas o recibos de nómina que demuestren el pago mensual por los servicios recibidos. Se aprecia variedad normativa.

En Asturias se exige la presentación de originales de las facturas emitidas durante el año, siempre justificando un importe igual o superior a la cuantía máxima. En Extremadura, se podrán exigir durante 
el periodo de prescripción y en cualquier momento las facturas y recibos bancarios, debiendo aportar el beneficiario el contrato de prestación de servicios, con indicación de fecha de inicio, horas de atención y tarifa del precio.

La Xunta de Galicia precisa que deben aportarse las facturas (duplicado), aceptándose como prueba los extractos bancarios o certificaciones, aunque si consiente el beneficiario lo puede efectuar directamente la entidad prestataria. De igual forma, certificación acreditativa del cumplimiento de las obligaciones de afiliación, alta y cotización a la Seguridad Social del asistente personal y, en el mes siguiente, copia del contrato suscrito. En La Rioja resulta suficiente la presentación de la copia que demuestre la relación contractual o certificación de la empresa que preste los servicios. En la Comunidad de Valencia se ha previsto un modelo de declaración responsable que debe suscribir quien asuma la asistencia personal.

La Consejería de Derechos Sociales en Navarra es muy exhaustiva en la justificación, estableciendo la obligación genérica de justificar el cumplimiento de los requisitos exigidos para la concesión, aunque de manera específica: presentar (en un mes desde la concesión) el contrato laboral (alta en Seguridad Social) o de prestación de servicios; comunicar las modificaciones; y justificar el gasto (nóminas y cotizaciones o facturación).

La Diputación Foral de Álava exige la presentación en el primer trimestre de cada anualidad de la facturación o recibos de salarios abonados en el año inmediatamente anterior. Para la comprobación de la afiliación, alta y cotizaciones en materia de Seguridad Social, se ha previsto, previa autorización de la persona beneficiaria, la consulta desde el instituto foral en colaboración con la Tesorería General de la Seguridad Social. Deberán presentarse ante el órgano público el contrato (precontrato, en su caso) y la titulación acreditativa (experiencia profesional, cuando proceda), sustituible por declaración jurada por el representante legal de la empresa de servicios, si fuese la modalidad elegida.

\subsection{Seguimiento del cumplimiento de requisitos y finalidad}

La normativa autonómica menciona frecuentes referencias a la práctica de los seguimientos por profesional de la entidad pública encargada del reconocimiento y justificación de requisitos (Cantabria, Castilla y León, Cataluña, La Rioja, Madrid, diputaciones forales de Álava, Gipuzkoa y Bizkaia). Esta actuación se incardina en la planificación de actividades del programa individual de atención, por medio de informe de evaluación (Cataluña). El seguimiento adquiere relevancia no solo para valorar la evolución del programa desde la perspectiva de la entidad pública, sino también para conocer el nivel de satisfacción de las atenciones recibidas (Benedicto, 2013).
Serán técnicos cualificados los encargados de efectuar el análisis de manera periódica (anual en la normativa de Cataluña y La Rioja). La obligación de colaboración se extiende no solo al beneficiario, sino también a los responsables de centros dedicados a la prestación de servicios (La Rioja).

La Diputación Foral de Álava precisa con detalle el alcance del seguimiento, determinando que debe incluir la verificación acerca de si el beneficiario conserva capacidad para tomar decisiones o se respetan las condiciones fijadas en el contrato. De igual modo, comprobar los apoyos, la intensidad, continuidad y calidad de los servicios prestados, así como el mantenimiento de la contratación, el cumplimiento de las obligaciones en materia de Seguridad Social y gastos producidos. Finalmente, la residencia efectiva en el territorio histórico de Álava (ocho meses al año) si la prestación se enmarca en el nivel adicional de protección. La normativa de la Diputación Foral de Gipuzkoa delega el seguimiento en los ayuntamientos, previa financiación. En la Diputación Foral de Bizkaia se individualiza cada expediente al identificarse con una persona coordinadora.

\subsection{Regulaciones específicas autonómicas}

En este apartado se recogen respuestas aportadas por las comunidades autónomas a problemas de diferente naturaleza planteados en la actividad gestora, normalmente circunstancias sobrevenidas en los protagonistas de la relación jurídica.

La Diputación Foral de Álava regula la situación de baja por incapacidad temporal del asistente personal, entendiendo que si no se contrata a un sustituto, la prestación permanece suspensa a partir del decimosexto día, siempre que no se prolongue más de seis meses, supuesto de extinción. La Diputación Foral de Gipuzkoa resuelve que se mantienen los requisitos con motivo de incapacidad temporal durante un periodo que comprende los días que resten hasta final de mes y los dos meses siguientes.

Para Extremadura, el ingreso temporal de la persona en situación de dependencia en un centro sanitario implica la suspensión de la prestación, debiendo comunicar fecha de ingreso y alta en el plazo de diez días. La Diputación Foral de Gipuzkoa entiende que continúan cumpliéndose los requisitos durante el periodo de vacaciones, hasta un máximo de treinta días por cada año natural.

\section{Conclusiones}

En los últimos años se aprecia un impulso decidido en varias comunidades autónomas dirigido a potenciar la prestación económica de asistencia personal, escasamente desarrollada en el sistema de dependencia, salvo excepciones (País Vasco). 
Una muestra de la nueva dinámica es la aprobación en varias comunidades autónomas de normativa exclusiva que profundiza en los contenidos más relevantes, incluyendo procesos de participación ciudadana en el diseño del modelo (Cataluña). Las aportaciones efectuadas por el Movimiento de Vida Independiente están contribuyendo al nuevo desarrollo.

Tras el análisis reglamentario autonómico, se detecta que el futuro de la asistencia personal debe centrarse en dos elementos relevantes: por un lado, una mayor dotación de la prestación económica; por otro, la aplicación de un modelo de compatibilidad más flexible, en el marco de un paquete de prestaciones.

Son varias las comunidades autónomas que reconocen un importe más elevado que el común estatal, en lógica con cargo al nivel propio de financiación (adicional). Dada la finalidad de contribuir a la contratación de una persona asistente personal (relación laboral, entidad social, empresa de servicios o empresario autónomo), se estima imprescindible incrementar los importes, toda vez que son cantidades máximas sometidas a reducción en función de la capacidad económica y la intensidad de los apoyos, además de deducciones derivadas de la percepción de complementos de análoga naturaleza y finalidad.

El segundo elemento susceptible de profundizar en su contenido es el modelo de compatibilidad. Como regla general, la compatibilidad es posible con la prevención, promoción de la autonomía personal y teleasistencia. La norma estatal establece varias posibilidades, algunas plasmadas en el desarrollo autonómico (ayuda a domicilio, centro de día/ noche, residencial parcial o temporal). Sin embargo, será el nivel de financiación propio el que permita mayor creatividad. ¿En qué sentido? Posiblemente la respuesta se enfoque en dirección a un paquete de prestaciones, recogido en el programa individual de atención tras análisis del caso concreto, que permita compatibilizar la asistencia personal con otros programas en función de la situación de necesidad y ponderando la intensidad a recibir por cada uno. 
AUDIENCIA NACIONAL (SALA DE LO CONTENCIOSO) (2011): Sentencia de 25 de febrero de 2011, recurso 90/2009.

BAÑEZ, T, y COMAS, D. (2021): “El asistente personal como trabajador/a: su implantación en España”, Zerbitzuan, no 74, pp. 99-113, 〈https://doi. org/10.5569/1134-7147.74.06).

BENEDICTO, Z. (2013): “La prestación económica de asistencia personal en el territorio histórico de Gipuzkoa", Zerbitzuan, no 54 , pp. 67-84, 〈https://doi.org/10.5569/1134-7147.54.04〉.

CARBONELL, G. J.: (2017): “El asistente personal para una vida independiente: una figura en construcción”, Revista de Derecho UNED, nํㅜ 21, pp. 187-216.

ESPAÑA (1982): Ley 13/82, de 7 de abril, de Integración Social de los Minusválidos (LISMI)", Boletín Oficial del Estado, no-103, 30-41982, «https://www.boe.es/buscar/doc. php?id=BOE-A-1982-9983>.

- (2006): Ley de Promoción de la Autonomía Personal y Atención a las personas en situación de dependencia, ley 39/2006, de 14 de diciembre, publicada en el Boletín Oficial del Estado, $\mathrm{n}$ 음 299, 15-12-2006, <https://www.boe.es/buscar/ act.php?id=BOE-A-2006-21990〉.

- (2008): Resolución de 2 de diciembre de 2008, de la Secretaría de Estado de Política Social, Familias y Atención a la Dependencia y la Discapacidad, por la que se publica el acuerdo del Consejo Territorial del Sistema para la Autonomía y Atención a la Dependencia, sobre criterios comunes de acreditación para garantizar la calidad de los centros y servicios del Sistema para la Autonomía y Atención a la Dependencia, Boletín Oficial del Estado, no 303, 17-12-
2008, 〈https://www.boe.es/buscar/doc. php?id=BOE-A-2008-20451>

- (2012): Real Decreto Ley 20/2012, de 13 de julio, sobre medidas para garantizar la estabilidad presupuestaria y de fomento de la competitividad, Boletín Oficial del Estado, no 168, 14-7-2012, <https://www.boe.es/buscar/ doc.php?id=BOE-A-2012-9364>.

- (2012): Resolución de 13 de julio de 2012, de la Secretaría de Estado de Servicios Sociales e Igualdad, por la que se publica el Acuerdo del Consejo Territorial del Sistema para la Autonomía y Atención a la Dependencia para la mejora del sistema para la autonomía y atención a la dependencia, Boletín Oficial del Estado, nํㅜ 185, 3-8-2012, 〈https://www.boe.es/buscar/doc. php?id=BOE-A-2012-10468>.

- (2013): Real Decreto 1051/2013, de 27 de diciembre de 2013, por el que se regulan las prestaciones del Sistema para la Autonomía y Atención a la Dependencia establecidas en la Ley 39/2006, n- -168 , Boletín Oficial del Estado, no -313 , 3112-2013, <https://www.boe.es/buscar/doc. php?id=BOE-A-2013-13811>.

- (2014): Resolución de 31 de julio de 2014, de la Secretaría de Estado de Servicios Sociales e Igualdad, por la que se publica el Acuerdo del Consejo Territorial de Servicios Sociales y del Sistema para la Autonomía y Atención a la Dependencia, sobre los criterios para determinar el contenido del servicio de promoción de la autonomía personal para las personas reconocidas en situación de dependencia en grado II y III, y la evaluación anual correspondiente al ejercicio 2013 de los resultados de aplicación de la Ley 39/2006, de 14 de diciembre, de Promoción de la Autonomía Personal 
y atención a las personas en situación de dependencia, Boletín Oficial del Estado, n- 197, 14-8-2014, 〈https://www.boe.es/buscar/doc. php?id=BOE-A-2014-8733'.

- (2015): Resolución de 3 de noviembre de 2015, de la Secretaría de Estado de Servicios Sociales e Igualdad, por la que se publica el Acuerdo del Consejo Territorial de Servicios Sociales y del Sistema para la Autonomía y Atención a la Dependencia, que modifica parcialmente el Acuerdo de 27 de noviembre de 2008 , sobre acreditación de centros y servicios del Sistema para la Autonomía y Atención a la Dependencia, Boletín Oficial del Estado, ํㅜ 274, 16-11-2015.

- (2017): Resolución de 11 de diciembre de 2017, de la Secretaría de Estado de Servicios Sociales e Igualdad, por la que se publica el Acuerdo del Consejo Territorial de Servicios Sociales y del Sistema para la Autonomía y Atención a la Dependencia, que modifica parcialmente el Acuerdo de 27 de noviembre de 2008 , sobre criterios comunes de acreditación para garantizar la calidad de los centros y servicios del Sistema para la Autonomía y Atención a la Dependencia, Boletín Oficial del Estado, n- 317, 30-12-2017.

- (2020): Resolución de 23 de marzo de 2020, de la Secretaría de Estado de Derechos Sociales, por la que se publica el Acuerdo del Consejo Territorial de Servicios Sociales y del Sistema para la Autonomía y Atención a la Dependencia, que modifica parcialmente el Acuerdo de 27 de noviembre de 2008 , sobre criterios comunes de acreditación para garantizar la calidad de los centros y servicios del Sistema para la Autonomía y Atención a la Dependencia", Boletín Oficial del Estado, no 82, 25-3-2020.

- (2021): Ley 8/2021, de 2 de junio, por la que se reforma la legislación civil y procesal para el apoyo a las personas con discapacidad en el ejercicio de su capacidad jurídica, Boletín Oficial del Estado, no 132, 3-6-2021, 〈https://www.boe.es/boe/ dias/2021/06/03/pdfs/BOE-A-2021-9233.pdf〉.

FARIÑAS, C. (2018): "El asistente personal, figura clave en la autonomía de la persona con dependencia", Revista Autonomía Personal, no 22, pp. 56-61.

FORO DE VIDA INDEPENDIENTE Y DIVERTAD (2015): “La asistencia personal. Características y acciones a tomar para su implantación”, Foro de Vida Independiente y Divertad, «http:// forovidaindependiente.org/la-asistenciapersonal-caracteristicas-y-acciones-a-tomarpara-su-implantacion/>.

GÓMEZ, N.; ORDAX, E.; MOYA, M.J. y SÁNCHEZ, F. (2015): "Prestación del servicio de asistencia personal”, en ORTEGA, E. (coord.), Situación de la asistencia personal en España, Plataforma Representativa Estatal de Personas con Discapacidad Física, pp. 26-34. 〈https://www. asistenciapersonal.org/informe.pdf〉.

JIMENO, F. (2017): “La acreditación de centros destinados a personas mayores en situación de dependencia: un instrumento al servicio del empleo y la calidad asistencial", e-Revista Internacional de la Protección Social, vol. II, no 2, pp. 114-127.

- (2018): “El impacto en las prestaciones económicas del sistema de dependencia español de los complementos deducibles de idéntica naturaleza y finalidad", e-Revista Internacional de la Protección Social, vol. III, nำ1, pp. 202214.

- (2021): "La coordinación de las prestaciones de asistencia personal en el Derecho de la Unión Europea", e-Revista Internacional de la Protección Social, vol. VI, no 1 , pp. 79-95.

IMSERSO (s.f.): “Información estadística destacada del SAAD", Instituto de Mayores y Servicios Sociales, 〈https://www.imserso.es/ imserso_01/documentacion/estadisticas/ info_d $/>$.

LA VANGUARDIA (2020): “La Generalitat impulsa un nuevo modelo de asistencia personal para personas con discapacidad física y sordoceguera", La Vanguardia, 8 de agosto, 〈https:// www.lavanguardia.com/local/catalunya/ catalunya-futur/20190808/463928632592/ nuevo-modelo-asistencia-personal-personasdiscapacidad-dependencia.html?.

MALDONADO, J.A. (2015): “Configuración jurídica de la figura del asistente personal", en ORTEGA, E. (coord.), Situación de la asistencia personal en España, Plataforma Representativa Estatal de Personas con Discapacidad Física, pp. 36-44, 〈https://www.asistenciapersonal.org/informe. pdf $>$.

ONU (2006): Convención sobre los Derechos de las Personas con Discapacidad, Nueva York, Organización de las Naciones Unidas, 13 de diciembre [instrumento de ratificación publicado en el Boletín Oficial del Estado, no 96, de 214-2008, «https://www.boe.es/buscar/doc. php?id=BOE-A-20086963'].

QUEZADA, M.Y. у MOTOS, C. (2017): Medición comparativa del impacto socioeconómico y presupuestario de la asistencia personal frente a otras prestaciones tradicionales de atención a la situación de dependencia, Observatorio Estatal de la Discapacidad, <https:// observatoriodeladiscapacidad.info/ wp-content/uploads/2018/09/MEDICIONIMPACTO-SOCIOECONOMICO-AP.pdf .

RODRÍGUEZ-PICAVEA, A. (2007): "Reflexiones en torno a la figura del asistente personal para la vida independiente y la promoción de la autonomía de las personas con diversidad funcional (discapacidad)", Zerbitzuan, no41, pp. 115-127.

SAAVEDRA, M. (2018): “La figura del asistente personal en la Ley 39/2006, de Promoción de la Autonomía y Atención a las personas en situación de dependencia ¿Realidad o quimera?", Aposta Revista de Ciencias Sociales, no 79, pp. 181-196.

SUÑE, A. y MARTÍNEZ, I. (2015): “La figura del asistente personal", en ORTEGA, E. (coord.), Situación de la asistencia personal en España, Plataforma Representativa Estatal de Personas con Discapacidad Física, pp. 18-23, 〈https://www. asistenciapersonal.org/informe.pdf〉.

TRIBUNAL SUPREMO (SALA DE LO SOCIAL, SECCIÓN $4^{\stackrel{a}{ })}$ (2012): Sentencia de 26 de noviembre de 2012. Normativa autonómica que desarrolla la prestación económica de asistencia personal. 
NORMATIVA AUTONÓMICA

QUE DESARROLLA LA PRESTACIÓN

ECONÓMICA DE ASISTENCIA PERSONAL

ANDALUCÍA (2007): Orden de 3 de agosto de 2007 por la que se establecen la intensidad de protección de los servicios, el régimen de compatibilidad de las Prestaciones y la Gestión de las Prestaciones Económicas del Sistema de Autonomía y Atención a la Dependencia en Andalucía, Boletín Oficial de la Junta de Andalucía, 16-8-2007.

ARAGÓN (2013): Orden de 24 de julio de 2013, de Aragón, del Departamento de Sanidad, Bienestar Social y Familia, por la que se regulan las prestaciones del Sistema para la Autonomía y Atención a la Dependencia, la capacidad económica de los beneficiarios y su participación en el coste de los servicios, en la Comunidad Autónoma de Aragón, Boletín Oficial de Aragón, 20-8-2013.

- (2017): Orden CDS 960/2017, de 26 de junio, por la que se modifica la Orden de 24 de julio de 2013 , del Departamento de Sanidad, Bienestar Social y Familia, por la que se regulan las prestaciones del Sistema para la Autonomía y Atención a la Dependencia, la capacidad económica de los beneficiarios y su participación en el coste de los servicios en la Comunidad Autónoma de Aragón, Boletín Oficial de Aragón, 12-7-2017.

CANARIAS (2019): Ley 16/2019, de 2 de mayo de 2019, de Servicios Sociales de Canarias, Boletín Oficial de Canarias, 17-5-2019.

CANTABRIA (2009): Orden EMP 48/2009, de 24 de abril, por la que se desarrolla el catálogo de servicios del sistema para la autonomía personal y la atención a la dependencia y se regula la aportación económica de las personas usuarias en la Comunidad Autónoma de Cantabria, Boletín Oficial de la Comunidad Autónoma de Cantabria, 29-4-2009.

CASTILLA Y LEÓN (2018): Orden FAM 6/2018, de 11 de enero, por la que se regulan las prestaciones del Sistema para la Autonomía y Atención a la Dependencia en Castilla y León, el cálculo de la capacidad económica y las medidas de apoyo a las personas cuidadores no profesionales, Boletín Oficial de Castilla y León, 7-1-2018.

- (2019): Orden FAM/806/2019, de 13 de septiembre, por la que se modifica la Orden FAM/6/2018, de 11 de enero, por la que se regulan las prestaciones del sistema para la autonomía y atención a la dependencia en Castilla y León, el cálculo de la capacidad económica y las medidas de apoyo a las personas cuidadoras no profesionales, Boletín Oficial de Castilla y León, 19-9-2019.

- (2021): Orden FAM 1/2021, de 4 de enero, por la que se modifica la Orden FAM/6/2018, de 11 de enero, por la que se regulan las prestaciones del Sistema para la Autonomía y Atención a la Dependencia en Castilla y León, el cálculo de la capacidad económica y las medidas de apoyo a las personas cuidadoras no profesionales, Boletín Oficial de Castilla y León, 14-1-2021

CASTILLA-LA MANCHA (2016): Decreto 3/2016, de 26 de enero, por el que se establece el catálogo de servicios y prestaciones económicas del Sistema para la Autonomía y Atención a la
Dependencia en la Comunidad Autónoma de Castilla-La Mancha y se determina la intensidad de los servicios y el régimen de compatibilidades aplicable, Diario Oficial de Castilla-La Mancha, 29-1-2016.

- (2019): Decreto 1/2019, de 8 de enero, del procedimiento para el reconocimiento de la situación de dependencia y del derecho de acceso a los servicios y prestaciones económicas del sistema para la autonomía y atención a la dependencia en Castilla-La Mancha, Diario Oficial de Castilla-La Mancha, 16-1-2019.

CATALUÑA (2010): Orden ASC/471/2010, de 28 de septiembre, por la que se regulan las prestaciones y los y las profesionales de la asistencia personal en Cataluña, Diari Oficial de la Generalitat de Catalunya, $\mathrm{n}$ - 5731, 8-10-2010.

COMUNIDAD DE MADRID (2015): Decreto 54/2015, de 21 de mayo, por el que se regula el procedimiento para reconocer la situación de dependencia y el derecho a las prestaciones del sistema para la autonomía y atención a la dependencia en la Comunidad de Madrid, Boletín Oficial de la Comunidad de Madrid, 26-5-2015.

COMUNIDAD FORAL DE NAVARRA (2019): Orden Foral 224/2019, de 31 de mayo, del Consejero de Derechos Sociales, por la que se regula la prestación económica para asistencia personal de las personas que tengan reconocida una situación de dependencia y/o discapacidad, Boletín Oficial de Navarra, 5-7-2019.

COMUNIDAD VALENCIANA (2017): Decreto 62/2017, de 19 de mayo, del Consell, por el que se establece el procedimiento para reconocer el grado de dependencia a las personas y el acceso al sistema público de servicios y prestaciones económicas, Diari Oficial de la Generalitat Valenciana, 13-6-2017.

DIPUTACIÓN FORAL DE ÁLAVA (2014): Decreto Foral 36/2014, del Consejo de Diputados, de 22 de julio, que regula el procedimiento para el reconocimiento de la situación de dependencia, la calificación de discapacidad, la existencia de trastornos del desarrollo o del riesgo de padecerlo y el derecho y procedimiento de acceso en tales condiciones a los servicios y prestaciones económicas de Servicios Sociales en Álava, Boletín Oficial del Territorio Histórico de Álava, 1-9-2014.

- (2018): Decreto Foral 5/2018, del Consejo de Gobierno Foral, de 30 de enero. Aprobar la actualización de las cuantías máximas desde el 1 de enero de 2018 de las prestaciones económicas del Sistema para la Autonomía y Atención a la Dependencia (SAAD) en Álava: prestación económica para cuidados en el entorno familiar y apoyo a cuidadores no profesionales, prestación económica de asistencia personal y prestación económica vinculada al servicio, Boletín Oficial del Territorio Histórico de Álava, 12-2-2018.

DIPUTACIÓN FORAL DE BIZKAIA (2013): Decreto Foral de la Diputación Foral de Bizkaia 103/2013, de 23 de julio, por el que se regula la prestación económica de asistencia personal, Boletín Oficial de Bizkaia, 26-7-2013.

- (2015): Decreto Foral de la Diputación Foral de Bizkaia 44/2015, de 17 de marzo, por el que se modifica 
el Decreto Foral 103/2013, de 23 de julio, por el que se regula la prestación económica de asistencia personal, Boletín Oficial de Bizkaia, 23-3-2015.

- (2015): Decreto Foral de la Diputación Foral de Bizkaia $85 / 2015$, de 23 de junio, por el que se aprueba en el Territorio Histórico de Bizkaia, las cuantías máximas para 2015, de la prestación económica para cuidados en el entorno familiar, asistencia personal y vinculada al servicio foral residencial, Boletín Oficial de Bizkaia, 26-6-2015.

DIPUTACIÓN FORAL DE GIPUZKOA (2017): Decreto Foral 24/2017, de 12 de diciembre, por el que se regulan las prestaciones económicas de la Ley 39/2006, de 14 de diciembre, de Promoción de la Autonomía Personal y Atención a las personas en situación de dependencia, Boletín Oficial de Gipuzkoa, 28-12-2017.

ESPAÑA (2007): Orden TAS/2455/2007, de 7 de agosto, por la que se dictan normas para la aplicación y desarrollo de los Reales Decretos que desarrollan la Ley 39/2006, de 14 de diciembre, de Promoción de la Autonomía Personal y Atención a las personas en situación de dependencia, en las Ciudades de Ceuta y de Melilla, Boletín Oficial del Estado, 10-8-2007.

EXTREMADURA (2012): Orden de 30 de noviembre de 2012 por la que se establece el catálogo de servicios y prestaciones económicas del Sistema para la Autonomía y Atención a la Dependencia, la intensidad de los servicios y el régimen de compatibilidades, aplicables en la Comunidad Autónoma de Extremadura, Diario Oficial de Extremadura, 5-12-2012.

- (2013): Orden de 7 de febrero de 2013 por la que se hacen públicos los criterios para la determinación, en la Comunidad Autónoma de Extremadura, de la prestación económica a los beneficiarios que tengan reconocida la condición de persona en situación de dependencia, Diario Oficial de Extremadura, 22-2-2013.

- (2017): Orden de 13 de noviembre de 2017 por la que se modifica la Orden de 30 de noviembre de 2012 por la que se establece el catálogo de servicios y prestaciones económicas del Sistema para la Autonomía y Atención a la Dependencia, la intensidad de los servicios y el régimen de compatibilidades, aplicables en la Comunidad Autónoma de Extremadura y la Orden de 7 de febrero de 2013 por la que se hacen públicos los criterios para la determinación, en la Comunidad Autónoma de Extremadura, de la prestación económica a los beneficiarios que tengan reconocida la condición de persona en situación de dependencia, Diario Oficial de Extremadura, 28-11-2017.

GALICIA (2013): Decreto 149/2013, de 5 de septiembre, por el que se define la cartera de servicios sociales para la promoción de la autonomía personal y la atención a las personas en situación de dependencia y se determina el sistema de participación de las personas usuarias en la financiación de su gasto, Diario Oficial de Galicia, 24-9-2013.

ILLES BALEARS (2010): Decreto 84/2010, de 25 de junio, por el que se regulan los criterios para calcular la capacidad económica con la finalidad de establecer la participación económica de las personas beneficiarias de las prestaciones asistenciales que forman parte de la Red Pública de Atención a la Dependencia de las Illes Balears, y para concretar las prestaciones económicas del Sistema para la Autonomía y Atención a la Dependencia, Boletín Oficial de las Islas Baleares, 3-7-2010.

LA RIOJA (2010): Orden 5/2010, de 30 de diciembre, de la Consejería de Servicios Sociales, por la que se regulan las condiciones de acceso y la cuantía de las prestaciones económicas del Sistema Riojano para la Autonomía Personal y la Dependencia, Boletín Oficial de La Rioja, 5-1-2011.

REGIÓN DE MURCIA (2010): Decreto 306/2010, de 3 de diciembre, por el que se establecen la intensidad de protección de los servicios, la cuantía de las prestaciones económicas, las condiciones de acceso y el régimen de compatibilidad de las prestaciones del sistema para la autonomía y atención a la dependencia en la Comunidad Autónoma de la Región de Murcia, Boletín Oficial de la Región de Murcia, 17-12-2010.

- (2016): Decreto-Ley 3/2016, de 1 de junio, por el que se modifica el régimen de participación económica de las personas beneficiarias de determinadas prestaciones del Sistema de Autonomía y Atención a la Dependencia en la Comunidad Autónoma de la Región de Murcia, Boletín Oficial de la Región de Murcia, 3-6-2016.

PRINCIPADO DE ASTURIAS (2015): Resolución de 30 de junio de $\mathbf{2 0 1 5}$, de la Consejería de Bienestar Social y Vivienda, por la que se regulan los servicios y las prestaciones económicas del sistema para la Autonomía y Atención a la Dependencia (SAAD) en el Principado de Asturias, Boletín Oficial del Principado de Asturias, 2-7-2015.

- (2019): Resolución de 24 de mayo de 2019, de la Consejería de Servicios y Derechos Sociales, por la que se establece un importe garantizado para las prestaciones económicas del sistema para la Autonomía y Atención a la Dependencia (SAAD) en el Principado de Asturias, Boletín Oficial del Principado de Asturias, 12-6-2019.

TRIBUNAL SUPERIOR DE JUSTICIA DE CATALUÑA (2012): Sentencia n$^{0}$ 598/2012, de 15 de octubre de 2012, recurso 418/2010. 